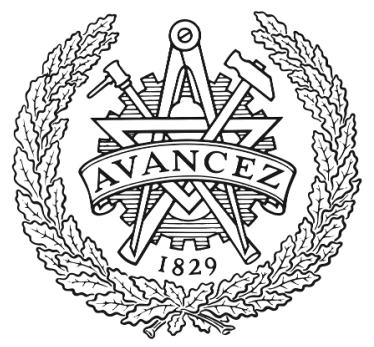

CHALMERS

UNIVERSITY OF TECHNOLOGY

\title{
Patterned Stimulation of Peripheral Nerves Produces Natural Sensations With Regards to Location but Not Quality
}

Downloaded from: https://research.chalmers.se, 2023-04-26 10:32 UTC

Citation for the original published paper (version of record):

Ortiz Catalan, M., Wessberg, J., Mastinu, E. et al (2019). Patterned Stimulation of Peripheral Nerves Produces Natural Sensations With Regards to Location but Not Quality. IEEE Transactions on Medical Robotics and Bionics, 1(3): 199-203. http://dx.doi.org/10.1109/TMRB.2019.2931758

N.B. When citing this work, cite the original published paper. 


\title{
Patterned stimulation of peripheral nerves produces natural sensations with regards to location but not quality
}

\author{
Max Ortiz-Catalan, Member, IEEE, Johan Wessberg, Enzo Mastinu, Student Member, IEEE, Autumn \\ Naber, Student Member, IEEE, and Rickard Brånemark
}

\begin{abstract}
Sensory feedback is crucial for dexterous manipulation and sense of ownership. Electrical stimulation of severed afferent fibers due to an amputation elicits referred sensations in the missing limb. However, these sensations are commonly reported with a concurrent "electric" or "tingling" character (paresthesia). In this study, we examined the effect of modulating different pulse parameters on the quality of perceived sensations. Three subjects with above-elbow amputation were implanted with cuff electrodes and stimulated with a train of pulses modulated in either amplitude, width, or frequency ("patterned stimulation"). Pulses were shaped using a slower carrier wave or via quasi-random generation. Subjects were asked to evaluate the natural quality of the resulting sensations using a numeric rating scale. We found that the location of the percepts was distally referred and somatotopically congruent, but their quality remained largely perceived as artificial despite employing patterned modulation. Sensations perceived as arising from the missing limb are intuitive and natural with respect to their location, and therefore useful for functional restoration. However, our results indicate that sensory transformation from paresthesia to natural qualia seems to require more than patterned stimulation.
\end{abstract}

Index Terms-neurostimulation, patterned stimulation, peripheral nerve stimulation, neuromusculoskeletal prostheses, cuff electrodes, prosthetic limbs, sensory feedback.

\section{INTRODUCTION}

$\mathrm{D}$ RECT nerve stimulation has the potential for providing intuitive, modality-matched, physiologically appropriate sensory feedback from artificial limbs. In 2014, two research groups provided the first demonstrations of the long-term viability of this approach [1], [2]. Tan et al. reported the stability of percepts using extraneural electrodes in two subjects for two years [1]. Independently, our group reported long-term stable percepts in one subject for over a year [2]. These studies provided supporting evidence for the safety and long-term stability of extraneural interfaces, as well as the physiologically appropriate location of percepts over time.

Extraneural [1]-[6] and intraneural [7]-[12] electrical stimulation of severed afferent nerves elicit sensations perceived as originating in the missing limb of amputees. Their

This study was funded by the Promobilia foundation, Swedish Research Council (Vetenskapsrådet), the Swedish Innovation Agency (VINNOVA), and the European Commission under the DeTOP project (LEIT-ICT-24-2015, Grant no. 687905).

M. Ortiz-Catalan, E. Mastinu, and A. Naber are with the Biomechatronics and Neurorehabilitation Laboratory (@ChalmersBNL), Department of distally referred localization, as well as their intuitive nature, makes these somatic sensations functionally valuable in prosthetic systems and natural in this regard. Sensations such as touch, pressure, and joint position (proprioception) have been reported by different groups [1], [2], [11], [3]-[10]. However, these familiar sensations are accompanied by an "electric" or "tingling" feeling, which turns their recognizable quality into an unnatural experience (paresthesia) [13]. Tan et al. remarked that delivering a train of pulses with fixed parameters does not correspond to the more natural, time-variant pulses a brain normally receives from biological sensors, and this may be the cause for a brain to deliver an experience qualitatively artificial in nature. They proposed to pattern the train of pulses by varying the width of each pulse in proportion to the amplitude of a slower modulating sinusoidal wave (Fig 1.A). This pulse width modulation (PWM) strategy was reported to transform the perception from "tingly" to "as natural as can be" [1]. They found this remarkable sensory transformation in two subjects implanted with five extraneural electrodes (two in the median and radial nerves, and one in the ulnar nerve). Percepts were elicited in different locations using up to 34 electrode contacts. Their subjects reported different sensations such as pulsing and constant pressure, vibration, tapping, and running on a texture [1]

Here, we report the use of such "patterned stimulation" technique in three transhumeral amputees implanted with a total of five cuff electrodes in median and ulnar nerves. We expanded our investigation to include pulse amplitude and frequency modulation, as well as quasi-random modulation on each stimulation modality. Whereas our findings are congruent with long-term stable distally referred sensations, our subjects reported that the percepts remained largely artificial in quality above perception threshold regardless of patterned stimulation.

\section{METHODS}

\section{A. Subjects}

Three unilateral, above-elbow amputees were recruited for this study. The subjects were implanted with cuff electrodes: three stimulation contacts per electrode; two and three

Electrical Engineering, Chalmers University of Technology, Gothenburg, Sweden.

J. Wessberg is with the Institute of Neuroscience and Physiology, University of Gothenburg, Gothenburg, Sweden.

R. Brånemark Department of Orthopaedics, Gothenburg University, Gothenburg, Sweden. 


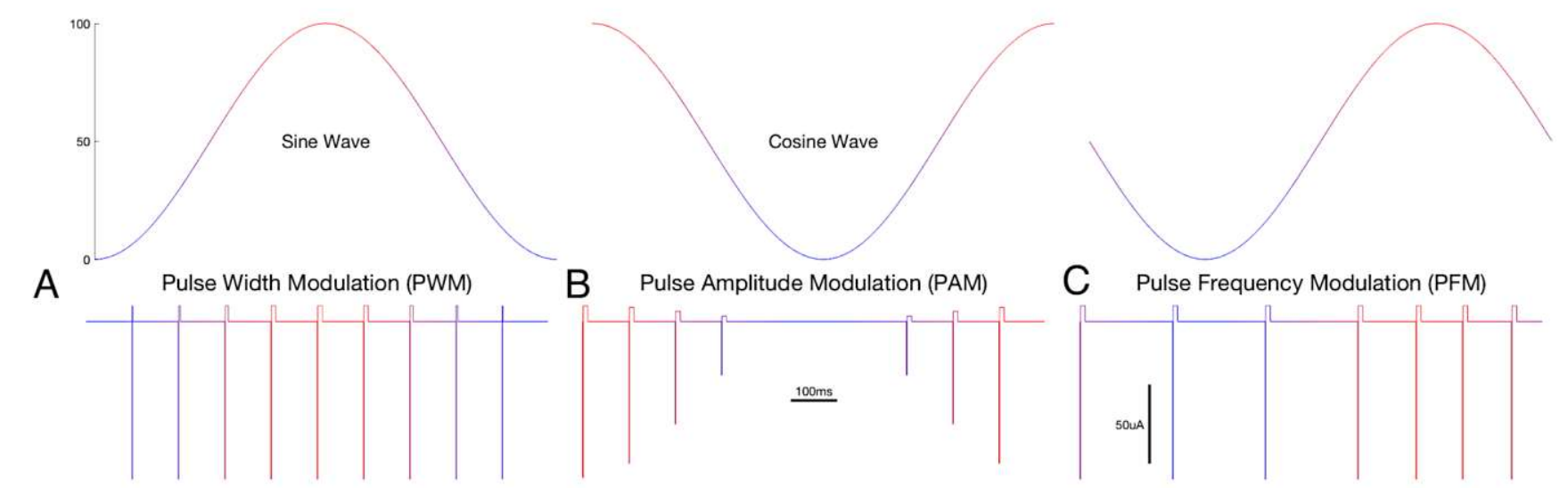

Fig. 1. Patterned stimulation. Modulating waves starting at $0 \%$ (sine), $100 \%$ (cosine), and $50 \%$ of maximal amplitude. The amplitude of the modulating wave dictates the width (A), amplitude (B), or frequency (C) of the stimulation pulses delivered to three amputees implanted with extraneural electrodes. A color code on the modulating waves and pulses illustrates how the peak value for the stimulation parameters is reached at the peak amplitude of the modulating wave. For illustration purposes, each modulating wave is presented patterning a different pulse parameter. The three modulating waves were used to pattern each of the stimulation parameters in the experiment (pulse width modulation - PWM, pulse amplitude modulation - PAM, and pulse frequency modulation - PFM).

electrodes in median and ulnar nerves, respectively; 15 stimulation contacts in total. Communication with the cuff electrodes was enabled via an osseointegrated interface that also provided direct skeletal attachment of the prosthesis [2]. Tests were conducted in subject 1 at $49-73$ months postimplantation, and 5-25 months post-implantation in subjects 2 \& 3. Subjects were blinded to the different stimulation paradigms, and these were presented in randomized order. This study was approved by the Västra Götalandsregionen ethical committee, and informed consent was obtained prior experimentation from all subjects.

\section{B. Patterned stimulation}

In the study by Tan et al., modulation of pulse width started from zero to peak amplitude in half the period of the modulating wave. We investigated PWM starting at $0 \%$ and $100 \%$ of the modulating wave (Fig 1.A-B). We utilized modulating frequencies of 1 and $4 \mathrm{~Hz}\left(f_{\mathrm{mod}}\right)$, and stimulation frequencies of 30 and $95 \mathrm{~Hz}\left(f_{\text {stim }}\right)$. We utilized the same square, chargebalanced, cathodic-first stimulation pulses as reported by Tan et al. [1]. Subjects received stimulations lasting two seconds and then reported the resulting perception.
In order to verify the concept of "patterned stimulation", we further investigated if modulation of pulse amplitude (PAM) or frequency (PFM) could achieve sensory transformation (Fig 1.B-C). We used stimulation parameters at magnitudes of the perception threshold of each condition, as any further increase of the modulated parameter resulted in sensation of higher intensity and more artificial quality. The peak (maximum) values for the modulated parameter were $100 \mu$ s and $200 \mu \mathrm{A}$ for PWM and PAM, respectively. Perception threshold were found by fixing the peak value of the modulated parameter and conducting an ascending staircase with the non-modulated parameter (steps of $10 \mu \mathrm{s}$ or $\mu \mathrm{A}$ ). Stimulation parameters are summarized using the standard suggested by Günter et al. [14] in TABLE I.

One can argue that modulation of a deterministic sinusoidal signal still provides a deterministic output. We therefore examined if arbitrary variations of pulse width, amplitude, or frequency led to a more natural sensation. The initial resolution of our neurostimulator (minimum step of $10 \mu \mathrm{s}$ and $\mu \mathrm{A}$ [15]) allowed us to construct quasi-random train pulses at higher $f_{\text {mod. }}$. We evaluate $f_{\text {mod }}$ from 5 to $9 \mathrm{~Hz}$ in steps of $1 \mathrm{~Hz}$ with $f_{\text {stim }}=30$ $\mathrm{Hz}$, and $f_{\text {mod }}$ from 10 to $100 \mathrm{~Hz}$ in steps of $10 \mathrm{~Hz}$ with $f_{\text {stim }}=95$

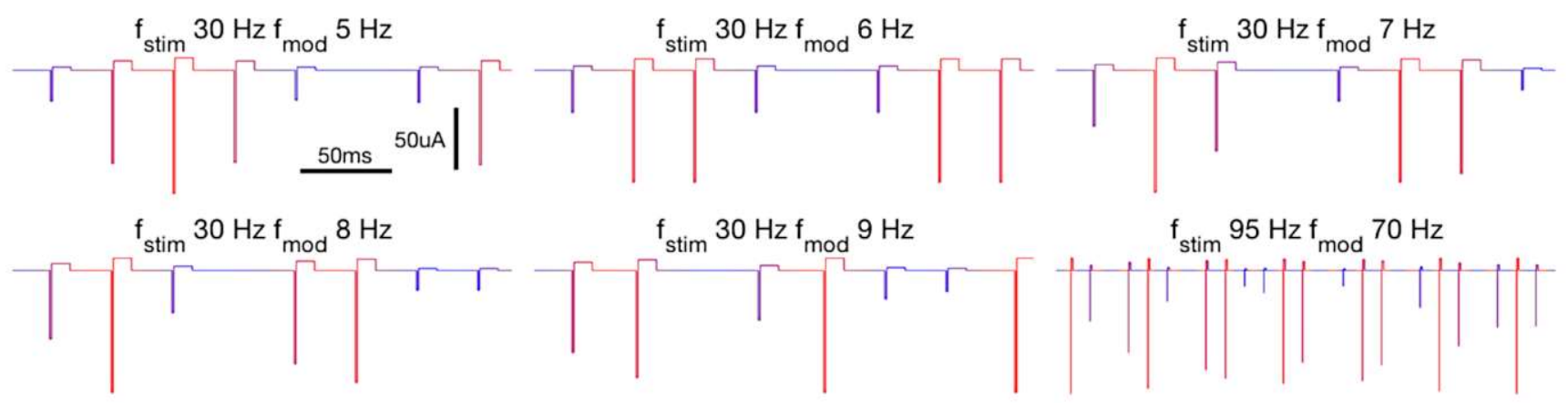

Fig. 2. Quasi-random stimulation pulses. Examples of quasi-random stimulation patterns used in this study. Quasi-random variations of pulse amplitude only are presented in the illustrations for clarity. These variations equally applied to pulse width and frequency. 

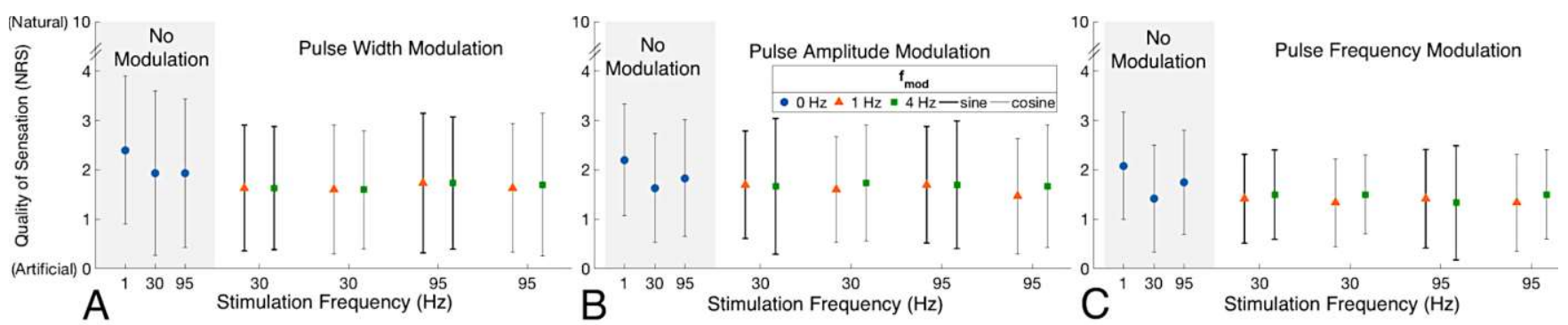

Fig. 3. Nature of elicited responses from pattern stimulation. Subjects evaluation on how "natural" the elicited sensations were perceived (Numeric Rating Scale: 0 -artificial and 10-natural). Stimulation was delivered by a single pulse, and a two-second train of pulses at $30 \mathrm{~Hz}$ and $95 \mathrm{~Hz}\left(f_{\text {stim }}\right)$ with no modulation, and PWM (A), PAM (B), and PFM (C) (modulation frequency $f_{\bmod }=1 \mathrm{~Hz}$ and $4 \mathrm{~Hz}$ ). Central markers represent the mean, and errors bars represent the standard deviation.

Hz. Examples of the resulting waveforms are shown in Fig. 2 for PAM. The variations in magnitude illustrated with PAM are analogous to the waveforms for PWM and PFM also employed in this study.

Sensory transformation from artificial to natural qualia was reported in ranges of up to $30 \mu \mathrm{S}$ using PWM [1], and therefore our original stimulation resolution of $10 \mu \mathrm{S}$ should suffice to achieve the effect. We nevertheless conducted an additional test using every PWM stimulation modality with a resolution of 1 $\mu \mathrm{S}$ as in the study by Tan et al. [1].

TABLE I

STIMULATION PARAMETERS [14]

\begin{tabular}{cl|cc}
\hline Label & \multicolumn{1}{c}{ Parameter } & Value & Unit \\
\hline A & Electrode surface area & 1.6 & $\mathrm{~mm}^{2}$ \\
D & $\begin{array}{l}\text { Duration of stimulating phase of a } \\
\text { stimulus pulse }\end{array}$ & $0-100$ & $\mu \mathrm{s}$ \\
& Duration of reversal phase of a & $10 * \mathrm{D}$ & $\mu \mathrm{s}$ \\
$\mathrm{D}^{\prime}$ & stimulus pulse & $1-100$ & $\mathrm{~Hz}$ \\
$\mathrm{~F}$ & Stimulation pulse frequency & $0-200$ & $\mu \mathrm{A}$ \\
$\mathrm{I}$ & Current of stimulating phase & $\mathrm{I} / 10$ & $\mu \mathrm{A}$ \\
$\mathrm{I}$ & Current of reversal phase & $2-200$ & - \\
$\mathrm{N}$ & Number of pulses per train & - & $\mathrm{Hz}$ \\
$\mathrm{R}$ & Train rate & 50 & $\mu \mathrm{s}$ \\
$\mathrm{W}$ & Inter-phase delay & $6-8$ & $\mathrm{Hours}$ \\
$\mathrm{P}$ & Total application time (per day) & $2-3$ & $\mathrm{Hours}$ \\
$\mathrm{S}$ & Effective stimulation time (per & $2.1-6.1$ & Years \\
$\mathrm{Y}$ & day) & &
\end{tabular}

\section{Evaluation of naturalness}

Whereas Tan et al. reported a qualitative dichotomy (artificial or natural), we asked our subjects to rate the natural quality of the resulting sensation using a numeric rating scale ( $\mathrm{NRS}_{\text {nat }}$ ). The $\mathrm{NRS}_{\text {nat }}$ lowest value "0" represented a completely artificial perception (such as "tingling/electric", or "touching a $9 \mathrm{~V}$ battery with the tongue"), and its highest value was " 10 ", representing a sensation identically as felt in their contralateral healthy hand or "as natural as can be". We defined "natural" to the subjects as a perceptual experience that could be elicited in their impaired contralateral hand, passively or actively interacting with an object or while freely moving (i.e., pressure, vibration, movement, etc.). Subjects also reported the intensity of the sensation using an NRS ( 0 no perception and 10 as strong as possible).

\section{RESULTS}

Subjects reported no change in the quality of the sensation from "artificial" to "natural" using patterned stimulation (Fig. 3A-C). Interestingly, a single pulse elicited the most natural sensation. This is arguably because the quality of the elicited sensations is difficult to discern at perception threshold due to its low intensity and brevity (a single pulse). However, as soon as a train of pulses was delivered, modulated or not, the sensations had a more electric character. As with sinusoidal modulation, the quality of the sensation with quasi-random pulse trains remained artificial in nature (Fig. 4).

At least in one occasion per subject, we found the same negative results by delivering simulation pulses with: $f_{\text {mod }}$ starting at $50 \%$ (Fig. 1.C); $f_{\bmod }$ at $0.5,2$, and $3 \mathrm{~Hz}$; restricted modulation ranges ("small-scale modulation" [1]) at $20 \%$ and $80 \%$; and stimulation resolution of $1 \mu \mathrm{S}$.

All percepts were reported in the expected areas innervated by the median and ulnar nerves. The quality of sensations was reported as "gentle touch" with an "electric" character. Such tactile quality has been reported in direct nerve stimulation at perception thresholds [6]. Subjects were able to track the intensity variations resulting from the modulating waveform by pressing on a force sensor as reported by Tan et al. [1]. In addition, we were able to confirm that 60 seconds of continuous stimulation do not result in a natural sensation as reported by Tan et al. [1], and contrary to the findings of Dhillon et al. [7].

\section{Discussion}

Stimulation parameters varied for different electrode contacts in the experiments by Tan et al. [1]. The peak pulse width (PW) increased until the subjects reported perception $\left(\mathrm{PW}_{\text {th }}\right)$. Increasing $\mathrm{PW}_{\text {th }}$ increased the intensity of the sensation until a given $\mathrm{PW}_{\text {tingle, }}$ where a "tingling" sensation appeared along with the original sensation. Beyond $\mathrm{PW}_{\text {tingle }}$, the sensation was eventually dominated by complete paresthesia at a given $\mathrm{PW}_{\text {mask. }}$ They found variable working ranges for $\mathrm{PW}$ that produced natural perception $\left(\mathrm{PW}_{\text {th }}<\mathrm{PW}_{\text {natural }}<\mathrm{PW}_{\text {tingle }}\right)$, and these ranges were reported to be up to approximately $30 \mu \mathrm{s}$. Our neurostimulator resolution was initially $10 \mu \mathrm{s}$, and therefore we expected to find the sensory transformation phenomena at least once. In addition, we monitored the intensity of each stimulation, which was reported at $1(91.5 \%)$ and $2(8.5 \%)$ 


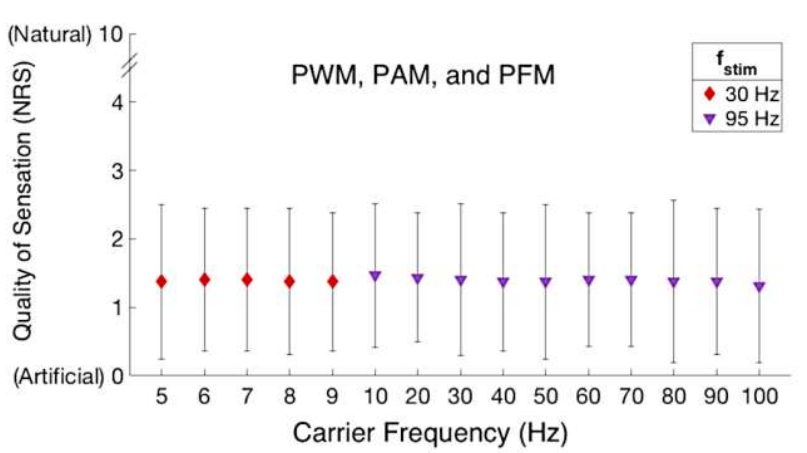

Fig. 4. Nature of elicited responses from quasi-random stimulation. Quasi-random variations were equally applied to pulse amplitude, width, and frequency. Quasi-random stimulation did not elicit a more "natural" perception. The only statistically significant differences were between a single pulse and the other conditions (Wilcoxon signed-rank test, $\mathrm{p}<0.01$ ). Central markers represent the mean, and errors bars represent the standard deviation.

NRS, which confirmed that we were at the lowest intensity levels where the sensory transformation was reported to appear $\left(\mathrm{PW}_{\text {th }}\right)$. Furthermore, we further verified our findings on at least one occasion, with each subject, using a resolution of $1 \mu \mathrm{S}$ in PWM, which resulted in the same outcome.

The highest $f_{\text {stim }}$ at which we could achieve fully reliable pattern stimulation in our hardware setup was $95 \mathrm{~Hz}$, whereas Tan et al. used predominantly $100 \mathrm{~Hz}$. Although relatively small, this difference could be a cause of the discrepancy. While capable of stimulating at higher fixed frequencies, our neurostimulator skipped 1 out of 10 pulses during pattern stimulation at $100 \mathrm{~Hz}$. We nevertheless tested this condition at least once on each subject, and we did not achieve sensory transformation to a more natural sensation either.

Whereas Tan et al. [1] used flat interface nerve electrodes (FINE) [16], we utilized spiral cuff electrodes [17]. These are both extraneural interfaces [18], and thus functionally equivalent at perception threshold for a single percept. FINE allows for higher selectivity by reshaping the nerve to bring all fascicles closer to the periphery. The difference with cuff electrodes is in the number of distinct percepts that can be achieved, not necessarily in the path followed by electric charges to elicit the smallest and weakest percepts (perception threshold). At perception threshold, the smallest possible amount of charge is used to elicit action potentials in one or a few afferent fibers. The closer the electrode is to the fibers, the smaller the amount of charge needed. The result is nevertheless the same; action potentials elicited in one or a few fibers. This rationale extends to intraneural electrodes as well, as we have confirmed that single percepts using cuff electrodes are equivalent to those found using single-unit intraneural stimulation [19].

\section{A. Implications on restoring function}

When an intact hand handles or touches an object, four different classes of mechanoreceptors are activated in different patterns and to varying degrees, depending on the contact location, shape, and structure (texture) of the object. Ideally, if we were to bypass the biological sensors and directly stimulate their corresponding afferent nerve fibers, we would need to interface each of those fibers, and be able to stimulate them differentially. For instance, in a steady grasp, mechanoreceptors with receptive fields at the highest-pressure points would be stimulated at higher frequencies than those in lower-pressure areas. This is technologically impossible at present, as we do not possess neural interfaces with such resolution. We are then left to blunt stimulation of a couple of neighboring fibers, which do not necessarily correspond to the same type of mechanoreceptor, thus making a truly biomimetic approach practically impossible at present. This lack of selectively is a fundamental problem in neural engineering that has not been addressed with enough resolution by current neural interfaces, including intraneural ones. Using multiple contacts could allow for steering current to stimulate different fiber populations [20], but these different populations would still be a mix of different physiological fiber types, and therefore such approach does not solve the selectivity problem with enough resolution. Okorokova et al. recognized the selectivity problem and proposed a neurostimulation model that simplifies the complexity of the tactile system when lacking selective stimulation [21]. Whereas such model increases sensory integration as intended [22], its admittedly simplified nature does not ensure that it would produce a natural experience. Fortunately, an exact replica of the biological system might not be necessary to restore function, as suggested by the success of cochlear implants.

One nevertheless must consider the complexity of the somatosensory system when contemplating that a substitute would produce numerically equivalent experiences (i.e., diversity of mechanoreceptors and their responses through different afferent fiber types). This complexity makes unlikely that patterned stimulation without discriminating fiber types, as employed here and by others [22], [23], would be capable to elicit a perceptual experience considered natural in the broader sense, that is, as an experience reproducible in an intact biological limb (obviously excluding artificial means such as direct nerve stimulation). The complexity of the somatosensory system seems to demand a solution for the aforementioned selectivity problem, which is arguably impossible to overcome using electrical stimulation [24]. In science, conservative hypotheses or explanations agree with the current state of knowledge. Our reasoning is therefore conservative, as it follows current knowledge of neurophysiology, laying out what would be require for true biomimicry (i.e., selective activation of different fiber types).

Regardless of the degree of qualitative "naturalness", sensations elicited via direct nerve stimulation still appear in somatotopically appropriate referred locations, and at variable intensities, which for example, could be used to mediate force during grasping without additional cognitive burden. Therefore, one can still argue in favor of direct nerve stimulation as means to restore functional sensory feedback to amputees. Using osseointegration as means of direct skeletal attachment, and as a gateway for bidirectional communication between implanted neuromuscular electrodes and the prosthetic limb [2], we are 
currently investigating the functional benefits of sensory feedback via direct nerve stimulation in daily life [25], and whether daily use changes the location and quality of the percepts. How stimulation must be performed in order to achieve a fully natural sensory experience is still an open question that requires further investigation and most likely, novel neural interfacing technologies.

\section{CONCLUSION}

Somatosensory feedback is relevant to the function and embodiment of an artificial device serving as replacement of a lost limb. Direct neural stimulation has been shown to be beneficial to both, although the qualitative experience does not yet equal those produced by a biological limb. We found that patterned stimulation did not produce a natural qualitative experience, arguably because fiber indiscriminate stimulation does not approach the biological mechanisms of sensory transfer. Lack of selectivity is a fundamental problem of neural interfaces preventing the differential stimulation of different afferent fiber types at appropriate resolution. Nevertheless, equaling qualia is not a prerequisite to equaling or enhancing function. Direct neural stimulation does produce long-term and intuitive distally referred sensation at graded intensities, which is theoretically enough to restore or enhance function.

\section{ACKNOWLEDGMENT}

We thank the subjects who participated in this study for their time and efforts. We also thank Sonam Iqbal for her contribution with the force tracking hardware, and Andrejs Litvins for his contribution to the firmware that enables higher resolution in the stimulation hardware.

\section{REFERENCES}

[1] D. W. Tan, M. A. Schiefer, M. W. Keith, J. R. Anderson, J. Tyler, and D. J. Tyler, "A neural interface provides longterm stable natural touch perception," Sci. Transl. Med., vol. 6, no. 257, p. 257ra138, Oct. 2014.

[2] M. Ortiz-Catalan, B. Håkansson, and R. Brånemark, “An osseointegrated human-machine gateway for long-term sensory feedback and motor control of artificial limbs," Sci. Transl. Med., vol. 6, no. 257re6, Oct. 2014.

[3] F. Clippinger, R. Avery, and B. Titus, "A sensory feedback system for an upper-limb amputation prosthesis.," Bull. Prosthet. Res., vol. 10-22, pp. 247-258, 1974.

[4] E. L. Graczyk, M. A. Schiefer, H. P. Saal, B. P. Delhaye, S. J. Bensmaia, and D. J. Tyler, "The neural basis of perceived intensity in natural and artificial touch," Sci. Transl. Med., vol. 8, no. 362, pp. 362ra142 LP-362ra142, 2016.

[5] M. A. Schiefer, E. L. Graczyk, S. M. Sidik, D. W. Tan, and D. J. Tyler, "Artificial tactile and proprioceptive feedback improves performance and confidence on object identification tasks," PLoS One, vol. 13, no. 12, p. e0207659, Dec. 2018.

[6] H. Charkhkar, C. E. Shell, P. D. Marasco, G. J. Pinault, D. J. Tyler, and R. J. Triolo, "High-density peripheral nerve cuffs restore natural sensation to individuals with lower-limb amputations," J. Neural Eng., vol. 15, no. 5, p. aac964, 2018.

[7] G. S. Dhillon, T. B. Krüger, J. S. Sandhu, and K. W. Horch, "Effects of short-term training on sensory and motor function in severed nerves of long-term human amputees.," J. Neurophysiol., vol. 93, no. 5, pp. 2625-33, May 2005.

[8] G. S. Dhillon and K. W. Horch, "Direct neural sensory feedback and control of a prosthetic arm.," IEEE Trans. Neural Syst. Rehabil. Eng., vol. 13, no. 4, pp. 468-72, Dec. 2005.

[9] S. Raspopovic, J. Carpaneto, E. Udina, X. Navarro, and S. Micera, "On the identification of sensory information from mixed nerves by using single-channel cuff electrodes.," $J$. Neuroeng. Rehabil., vol. 7, p. 17, Jan. 2010.

[10] F. M. Petrini et al., "Six-Month Assessment of a Hand Prosthesis with Intraneural Tactile Feedback," Ann. Neurol., vol. 85 , no. 1, pp. 137-154, Jan. 2019.

[11] T. S. Davis et al., "Restoring motor control and sensory feedback in people with upper extremity amputations using arrays of 96 microelectrodes implanted in the median and ulnar nerves," J. Neural Eng., vol. 13, no. 3, p. 036001, Jun. 2016.

[12] S. Wendelken et al., "Restoration of motor control and proprioceptive and cutaneous sensation in humans with prior upper-limb amputation via multiple Utah Slanted Electrode Arrays (USEAs) implanted in residual peripheral arm nerves," J. Neuroeng. Rehabil., vol. 14, no. 1, p. 121, Dec. 2017.

[13] Å. B. Vallbo, K. Å. Olsson, K.-G. Westberg, and F. J. Clark, "Microstimulation of Single Tactile Afferents From the Human Hand," Brain, vol. 107, no. 3, pp. 727-749, 1984.

[14] C. Günter, J. Delbeke, and M. Ortiz-Catalan, "Safety of long-term electrical peripheral nerve stimulation: review of the state of the art," J. Neuroeng. Rehabil., vol. 16, no. 1, p. 13, Dec. 2019.

[15] E. Mastinu, P. Doguet, Y. Botquin, B. Hakansson, and M. Ortiz-Catalan, "Embedded System for Prosthetic Control Using Implanted Neuromuscular Interfaces Accessed Via an Osseointegrated Implant," IEEE Trans. Biomed. Circuits Syst., vol. 11, no. 4, pp. 867-877, Aug. 2017.

[16] D. J. Tyler and D. M. Durand, "Functionally selective peripheral nerve stimulation with a flat interface nerve electrode," Neural Syst. Rehabil. Eng. IEEE Trans., vol. 10, no. 4, pp. 294-303, 2002.

[17] B. P. Christie et al., "Long-term stability of stimulating spiral nerve cuff electrodes on human peripheral nerves," $J$. Neuroeng. Rehabil., vol. 14, no. 1, p. 70, Dec. 2017.

[18] M. Ortiz-Catalan, R. Brånemark, B. Håkansson, and J. Delbeke, "On the viability of implantable electrodes for the natural control of artificial limbs: Review and discussion," Biomed. Eng. Online, vol. 11, 2012.

[19] R. Ackerley, H. Backlund Wasling, M. Ortiz-Catalan, R. Brånemark, and J. Wessberg, "Case Studies in Neuroscience: Sensations elicited and discrimination ability from nerve cuff stimulation in an amputee over time," $J$. Neurophysiol., vol. 120, no. 1, pp. 291-295, Jul. 2018.

[20] J. Sweeney, D. Ksienski, and J. T. Mortimer, "A nerve cuff technique for selective excitation of peripheral nerve trunk regions," IEEE Trans Biomed Eng, vol. 37, no. 7, pp. 706$715,1990$.

[21] E. V. Okorokova, Q. He, and S. J. Bensmaia, "Biomimetic encoding model for restoring touch in bionic hands through a nerve interface," J. Neural Eng., vol. 15, no. 6, p. 066033 , Dec. 2018.

[22] G. Valle et al., "Biomimetic Intraneural Sensory Feedback Enhances Sensation Naturalness, Tactile Sensitivity, and Manual Dexterity in a Bidirectional Prosthesis," Neuron, vol. 100, no. 1, pp. 37-45.e7, 2018.

[23] G. Valle et al., "Comparison of linear frequency and 
amplitude modulation for intraneural sensory feedback in bidirectional hand prostheses," Sci. Rep., vol. 8, no. 1, p. 16666, Dec. 2018.

[24] M. Ortiz-Catalan, "Restoration of somatosensory perception via electrical stimulation of peripheral nerves," Clin. Neurophysiol., vol. 129, no. 4, pp. 845-846, 2018.

[25] M. Ortiz-Catalan, E. Mastinu, R. Brånemark, and B. Håkansson, "Direct neural sensory feedback and control via osseointegration," in XVI World Congress of the International Society for Prosthetics and Orthotics (ISPO), 2017.

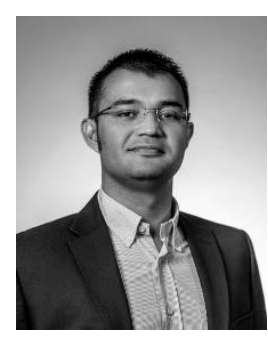

Max Ortiz Catalan (M'14) received the electronics engineering degree from the ITESM Campus Toluca, Mexico, in 2005, the M.Sc. degree in complex adaptive systems and the Ph.D. degree in biomedical engineering from Chalmers University of Technology, Gothenburg, Sweden, in 2009 and 2014, respectively.

$\mathrm{He}$ is currently an Assoc. Professor with the department of Electrical Engineering, Chalmers University of Technology, where he founded the Biomechatronics and Neurorehabilitation Laboratory. He also serves as the Research Director with Integrum AB, Sweden. His research interests include bioelectric signals acquisition electronics, signal processing and artificial intelligence algorithms for decoding motor volition and control, neural interfaces, neurostimulation, bone-anchored prostheses and osseointegration, virtual and augmented reality for neuromuscular rehabilitation and the treatment of neuropathic pain.

Dr. Ortiz Catalán has received several honors, notably the European Youth Award in 2014, the Delsys Prize in 2016, the Brian \& Joyce Blatchford Award in 2017, and the Swedish Embedded Award in 2018. He is an Associate Editor for the Journal of Neuroengineering and Rehabilitation (JNER) and the IEEE Transaction on Medical Robotics and Bionics (TMR-B).

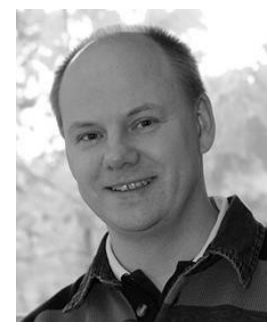

Johan Wessberg received the Ph.D. degree in physiology from the University of Gothenburg, Sweden, in 1995.

He is currently Professor in Physiology at the Institute of Neuroscience and Physiology, Sahlgrenska Academy, University of Gothenburg. His laboratory focuses on peripheral and central nervous system mechanisms of the human sense of touch, investigated using a combination of peripheral nerve recording from tactile receptors with the microneurographic technique, psychophysics, and functional brain imaging using fMRI, EEG and MEG. In 1999-2000 he was a research associate at Duke University, Durham, NC, USA in the laboratory of Prof. Miguel Nicolelis, where he pioneered work on a Brain-Machine Interfaces in monkeys using arrays of chronically implanted microelectrodes. Current research topics include pleasant touch to the skin, neural interfacing including prosthetic touch, and sensations and brain activity elicited by targeted stimulation of single tactile nerve fibers.

Prof. Wessberg is a member of the Society for Neuroscience (USA) and the Scandinavian Physiological Society.

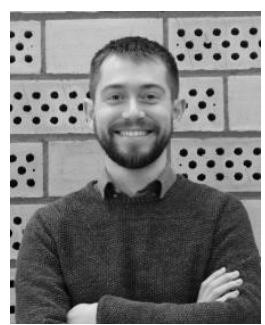

Enzo Mastinu received the M.Sc. degree with honors in electronic engineering from Universita' degli Studi di Cagliari (UNICA), Italy, in 2014 and the Ph.D. degree in biomedical engineering from Chalmers University of Technology, Sweden, in 2019.

$\mathrm{He}$ was $\mathrm{HW} / \mathrm{FW}$ designer for a spin-off company during his M.Sc. education, and then a postgraduate researcher for 6 months at UNICA. He is currently a post-doctoral researcher within the Biomechatronics and Neurorehabilitation Laboratory at Chalmers University of Technology. His research focuses on prosthetic control for upper limb amputees and the research interests cover bioelectric signal acquisition and processing, embedded systems and electronics design, myoelectric pattern recognition, as well as bone-anchored prostheses and sensory feedback via neural stimulation.

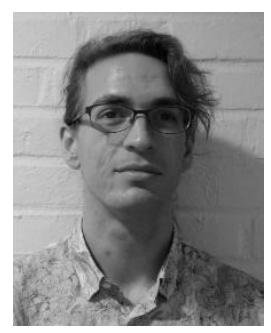

Autumn Naber received the B.Sc. degree in computer engineering from California State University, Sacramento, U.S.A. in 2013 and the M.Sc. degree in biomedical engineering from Chalmers University of Technology (CTH), Gothenburg, Sweden, in 2017.

She is currently working towards a Ph.D. in biomedical engineering at $\mathrm{CTH}$. Her current research focuses on embedded systems and materials for improving myoelectric control for people with severe neuromuscular disorders

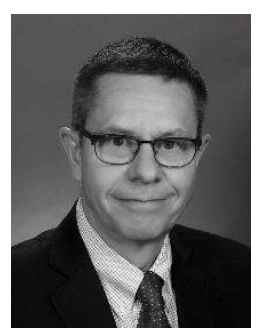

Rickard Brånemark received the M.D. and $\mathrm{Ph} . \mathrm{D}$. degrees in orthopedics from the University of Gothenburg, Sweden, in 1987 and 1996, respectively. He received the M.Sc. degree in technical physics from Chalmers University of Technology, Gothenburg, Sweden, in 1987.

He is presently Research Scientist at the Biomechatronics Group at MIT Media Lab, Cambridge, MA; Assoc. Prof. at the Dept. of Orthopaedics, Gothenburg University; Visiting Assoc. Prof. at Harvard Medical School, Boston, MA; Prof. Emeritus at the Dept. of Orthopaedics, University of California, San Francisco, CA; Chairman and Founder of Integrum AB, Sweden. He is one of the most experienced osseointegration orthopaedic surgeon in the world. He was the lead surgeon for the team that in 2013 treated a patient with an above elbow amputation with a combination of a percutaneous osseointegrated implant and implanted muscle and nerve electrodes. He has published more than 70 scientific peer-reviewed papers on different aspects of osseointegration ranging from material properties to in-depth patient interviews, with a focus on clinical outcomes, biomechanics and interface analysis.

Prof. Brånemark received the Hanger prize for his contribution to amputee treatment in 2007, the UCSF Presidential Chair Award in 2016, the Brian and Joyce Blatchford Award in 2017, the Per Uddén Prize in 2017, and the Henry Wallman prize 2019. 